\title{
Under-5 mortality due to HIV: regional levels and 1990-2009 trends
}

\author{
Karen Stanecki, ${ }^{1}$ Juliana Daher, ${ }^{1}$ John Stover, ${ }^{2}$ Priscilla Akwara, ${ }^{3}$ Mary Mahy ${ }^{1}$
}

'UNAIDS, Geneva, Switzerland

${ }^{2}$ The Futures Institute,

Glastonbury, Connecticut, USA

3UNICEF, New York, USA

\section{Correspondence to}

Karen Stanecki, UNAIDS, 20

Avenue Appia, 1211 Geneva,

27, Switzerland

staneckik@unaids.org

Accepted 7 October 2010

\section{ABSTRACT}

Background Since at least the late 1990s, HIV has been viewed as a major threat to efforts by countries to reduce under-5 mortality. Previous work has documented increased under-5 mortality due to HIV from 1990 to 1999 in Africa. The current analysis presents estimates and trends in under-5 mortality due to HIV in low- and middle-income countries by region up to 2009 .

Methods The analyses are based on the national models of HIV and AIDS produced by country teams in coordination with UNAIDS and its partners for the years 1990-2009. These models produce a time series of estimates of HIV-related mortality as well as overall mortality in children aged $<5$ years.

Results and conclusion These analyses indicate that, in 2009, HIV accounted for roughly 2.1\% (1.2-3.0\%) of under-5 deaths in low- and middle-income countries and $3.6 \%(2.0-5.0 \%)$ in sub-Saharan Africa. The percentage of under- 5 deaths due to HIV has been falling in the last decade-for example, from $2.6 \%(1.6-3.5 \%)$ in 2000 to $2.1 \%(1.2-3.0 \%)$ in 2009 in low- and middle-income countries and from $5.4 \%(3.3-7.3 \%)$ in 2000 to $3.6 \%$ $(2.0-5.0 \%)$ in 2009 in sub-Saharan Africa. This fall in the percentage of under-5 deaths due to HIV has been driven by a combination of factors including scale-up of prevention of mother-to-child transmission programmes and treatment for pregnant women and children as well as a decrease in the prevalence of HIV among pregnant women.

\section{INTRODUCTION}

In 2001 the global community came together in the United Nations special session on HIV/AIDS and agreed to a set of global targets in response to a rapidly escalating global public health crisis. ${ }^{1}$ While there were specific targets set for AIDS, there was also an acknowledgement of the impact that HIV and AIDS could have on the goals for child survival. Specifically, it was argued that increases in HIV-related mortality could make it difficult for many countries with a high prevalence of HIV to reach their goals for under- 5 mortality.

Previous work has investigated the relative importance of HIV as a cause of death among children at country and regional levels. ${ }^{2-4} \mathrm{~A}$ more recent analysis of the causes of child deaths found that, in 2008 , deaths due to HIV were the cause of $4 \%$ of child deaths in Africa. ${ }^{5}$ Another analysis of the trend in under-5 mortality found that overall mortality in children younger than 5 years has decreased from 11.9 million deaths in 1990 to 7.7 million deaths in 2010. ${ }^{6}$ Increasing coverage of HIV interventions has an impact on the number of children dying from HIV. These interventions include scale-up of programmes to prevent mother-to-child transmission of HIV
(PMTCT), antiretroviral therapy (ART) for children with HIV and ART for adults. All of these interventions have been scaled up in the last few years and all should have a significant impact on HIV-related deaths in children under the age of 5 years. ${ }^{78}$

In addition, there is increasing evidence that the prevalence of HIV in adults and in pregnant women has peaked and fallen in some of the countries with a higher HIV prevalence. These include countries such as Zimbabwe, ${ }^{9}$ Ethiopia, ${ }^{10}$ Zambia $^{11}$ and Kenya. ${ }^{12}$ These reductions in prevalence will result in fewer children being exposed to HIV via motherto-child transmission and could have a significant impact on overall under- 5 deaths due to HIV.

In this paper we use the most recent estimates of HIV to look at the overall trend in mortality due to HIV in children from 1990 to 2009. First we estimate the number of under- 5 deaths due to HIV by year from 1990 through 2009; second, we estimate the proportion of under-5 mortality due to HIV; and, third, we look at the relative impact of PMTCT, ART and falling prevalence on under- 5 mortality due to HIV. For all of these analyses we present estimates for low- and middle-income countries with a primary focus on sub-Saharan Africa.

\section{METHODS}

The national and global HIV estimation process began with country-specific estimates of HIV prevalence collected through biennial regional workshops and analysed using the Estimates and Projection Package (EPP), Workbook and Spectrum software packages whose methodology is described elsewhere. ${ }^{13-15}$ Briefly, for countries with a generalised epidemic, EPP fits a simple epidemiological model developed by the UNAIDS Reference Group on Estimates, Modelling and Projections to observed HIV surveillance data using a maximum likelihood method. For countries with a concentrated epidemic, HIV prevalence is estimated by combining the HIV prevalence in specific high- and low-risk population groups with an estimate of the size of these groups. For countries with enough trend data, EPP is used to fit a curve for the various high- and low-risk populations. For countries with limited data, the Workbook method is repeated for several years and then a prevalence trend is derived in EPP. For countries where a national household survey has collected HIV prevalence data, this information is used as well as the sentinel surveillance data to inform the estimates of adult prevalence and the male:female ratios.

Time trends of the incidence of HIV in adults (age 15-49 years) are then transferred to Spectrum and the data are entered for coverage of all PMTCT interventions (notably antiretroviral (ARV) agents 
for prophylaxis or treatment, infant feeding modes and duration), ART and child treatment for each year. Estimated HIV outcomes (eg, HIV incidence, HIV prevalence and mortality due to HIV by age and sex) are then produced using the AIDS Impact Model module. New infections in children are calculated as a proportion of total births. That proportion is the product of HIV prevalence among pregnant women in each age group and the mother-to-child transmission rate and is adjusted for the fertility of HIV-infected women. The transmission rate is determined by the treatment regimen (single, dual, triple or no therapy), type of feeding (mixed breast feeding, exclusive breast feeding, no breast feeding) and the length of breast feeding. ${ }^{14}$ Non-HIV-related mortality in children is determined by the estimates of life expectancy in the absence of AIDS from the UN Population Division 2008 revision and the appropriate model life table. HIV-related deaths in children are calculated based on the number of HIV-positive children, their rate of progression to death with and without ART and the effects of cotrimoxazole and ART on enhancing survival.

The Spectrum software tracks children separately for those infected during pregnancy and delivery (perinatal transmission) and those infected after birth through breast feeding. In both cases, survival by age follows a double Weibull distribution. ${ }^{16} 17$ For children infected at birth, the pattern shows $51 \%$ survival by age $1,39 \%$ by age 2 and $35 \%$ by age 5 . For children infected through breast feeding, the pattern shows $77 \%$ survival 1 year after infection, $65 \%$ after 2 years and $59 \%$ after 5 years.

The assumed perinatal transmission rates for different ARV regimens are as follows:

- No ARV: $20 \%$.

- Single-dose nevirapine: $11 \%$.

- Combination therapy using dual prophylaxis: $4 \%$.

- Combination therapy using triple prophylaxis ARV: $2 \%$ (there is also a separate input for mothers on ART for their own health with the same transmission rate).

HIV-exposed infants born HIV-negative (uninfected) have a monthly probability of HIV infection based on the infant feeding method and duration as well as use of ARV drugs during breast feeding. The assumed monthly transmission rates during breast feeding are estimated to be:

- Replacement feeding: $0 \%$.

- Mixed breast feeding during months 1-6: $1.5 \%$.

- Exclusive breast feeding during months 1-6: $0.75 \%$.

- Mixed breast feeding 6-36 months: 0.75\%.

- Breast feeding in the presence of maternal ART or effective prophylaxis: $0.3 \%$.
Spectrum projections from 117 low- and middle-income countries from sub-Saharan Africa, Latin America and the Caribbean, Eastern Europe and Asia were used in this study and all results throughout refer to these countries. HIV-related deaths in children aged $<5$ years as well as deaths due to all causes including HIV were extracted from Spectrum for each country and summed by region and globally. The proportion of under-5 mortality attributable to HIV was then calculated. Plausibility bounds for deaths due to HIV in children aged $<5$ years were calculated based on the uncertainty bounds for deaths due to HIV in children aged 0-14 years calculated through a Monte Carlo procedure that varies all key assumptions including adult HIV prevalence, the rate of mother-to-child transmission, the rate of progression and the effectiveness of treatment. Definitions of regions can be found on the UNAIDS website under AIDS Info. ${ }^{18}$

We also considered the relative impact of three interventions on mortality: declining HIV incidence among pregnant women, PMTCT programmes and child ART programmes. Using two country files with a recently declining prevalence of HIV among pregnant women (Botswana and Zimbabwe), several scenarios were developed to measure this impact. ${ }^{19} 20$ We used the basic EPP and Spectrum files to estimate HIV in each country and then modified the inputs. The following scenarios were developed varying only one parameter:

1. Changing declining incidence to stable incidence as of 2000 .

2. Removing all PMTCT programme data.

3. Removing all child ART coverage.

4. Removing all cotrimoxazole coverage for children exposed to HIV.

\section{RESULTS}

The number of deaths attributable to HIV in children aged $<5$ years increased from $69000(38000-110000)$ in 1990 to 260000 (160000-340000) in 2002 (the peak year) before declining to 170000 (100000-250000) in 2009 (figure 1). The proportion of deaths due to HIV among children aged $<5$ years of all deaths in children $<5$ years reached a peak in 2004 of $2.9 \%$ $(1.9-3.9 \%)$ and began to decline thereafter to $2.2 \%(1.3-3.1 \%)$ in 2009 .

In countries outside sub-Saharan Africa the proportion of deaths among children aged $<5$ years attributable to HIV peaked at $>0.4 \%$ in 2002 and has started to fall slightly since 2007 (figure 2).

In sub-Saharan Africa the proportion of deaths in children $<5$ years of age due to HIV reached a peak at $5.4 \%(3.4-7.4 \%)$ in
Figure 1 Number of deaths due to HIV in children aged $<5$ years in sub-Saharan Africa, Latin America and the Caribbean, Eastern Europe and Asia, 1990-2009.

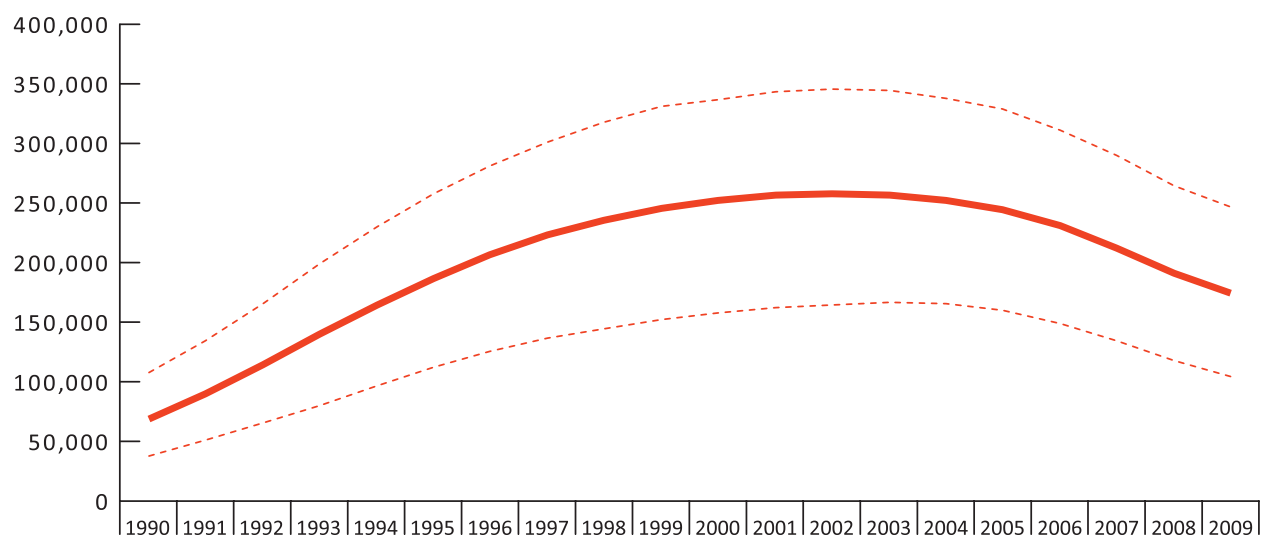


Figure 2 Proportion of under-5 mortality attributable to HIV in low- and middle-income countries in Latin America and the Caribbean, Eastern Europe and Asia, 1990-2009.

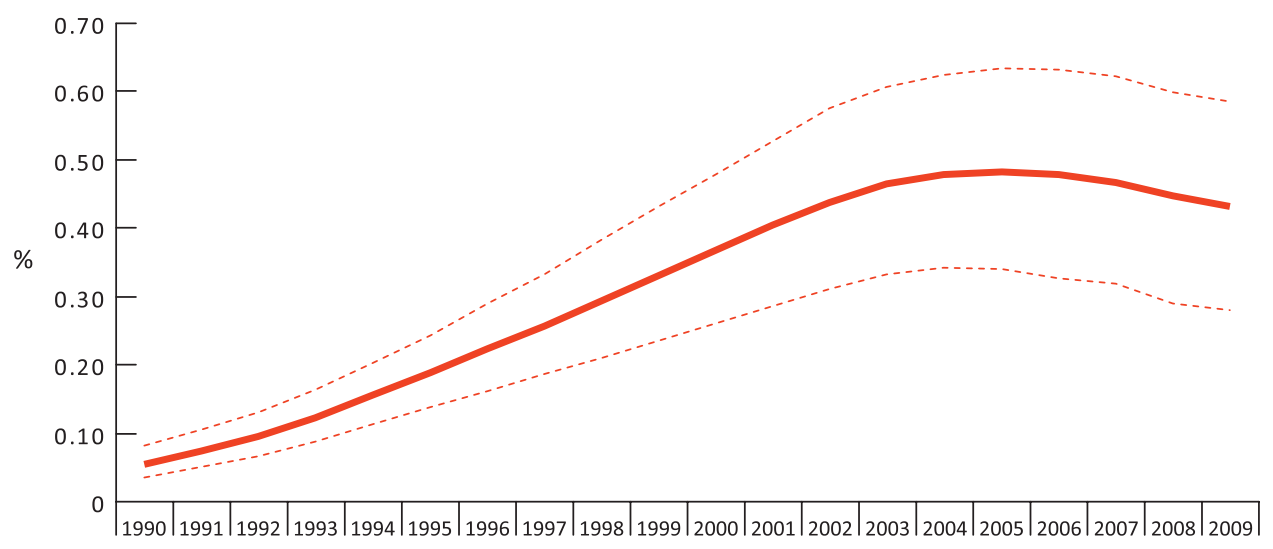

Estimate $\quad \ldots-\cdots$ High and low estimates
2002 and began to decline thereafter to $3.6 \%(2.0-5.0 \%)$ in 2009 (figure 3 ). This pattern varies by region in sub-Saharan Africa, as shown in figure 4.

The highest proportion of deaths among children due to HIV is seen in Southern Africa. In 1990 the proportion of deaths due to HIV among children aged $<5$ years was similar in Southern and Eastern Africa. However, that pattern changed, reflecting the rapidly increasing HIV epidemic in Southern Africa and, in most countries of that region, lower levels of under-5 mortality due to non-HIV-related causes. ${ }^{6}$ In 2003 an estimated $15.5 \%$ (11.4-19.1\%) of all deaths among children aged $<5$ years were due to HIV; by 2009 there was a decline of $>55 \%$ to $9.9 \%$ $(6.6-12.2 \%)$ in Southern Africa. In Central Africa the proportion of HIV-related deaths peaked in 2003 at $2.5 \%(1.3-3.6 \%)$ and declined to $2.0 \%(1.0-3.0 \%)$ in 2009. In Eastern Africa the proportion of HIV-related deaths among under-5 mortality peaked in 1999 at $6.5 \%(3.5-9.3 \%)$ and decreased to $3.6 \%$ $(1.5-6.2 \%)$ in 2009 , a decline of nearly $45 \%$. In Western Africa the proportion of under-5 deaths due to HIV declined from $2.9 \%$ $(1.5-4.2 \%)$ in 2000 to $2.3 \%(1.1-3.4 \%)$ in 2009.

In 2009 the number of HIV-related deaths in children aged $<5$ years in sub-Saharan Africa was highest in Nigeria $(>30000)$ and South Africa ( $>20000)$ (figure 5). Overall, Eastern Africa showed relatively high numbers of HIV-related deaths in children $<5$ years of age. In countries outside sub-Saharan Africa, the country with the highest estimated number of HIV-related deaths was India (7700 (3200-14000)). All other countries had fewer than 1000 HIV-related deaths in children aged $<5$ years.

The proportion of under- 5 deaths attributable to HIV was highest in countries in Southern Africa, including Lesotho, Namibia, South Africa, Swaziland and Zimbabwe (all $>20 \%$ ) in 2009 (figure 6). The proportion of under-5 deaths attributable to HIV in Botswana peaked at 48.2\% (27.5-63.0\%) in 2001 and declined to $16.0 \%(6.2-23.1 \%)$ in 2009 . Similarly, in Swaziland the proportion of under-5 deaths attributable to HIV peaked at $39.0 \%(24.3-50.1 \%)$ in 2005 but declined to $22.3 \%$ (11.0-34.0\%) in 2009. The proportion in South Africa declined from a peak of $38.0 \%(22.0-49.7 \%)$ in 2004 to $26.0 \%$ $(15.0-34.5 \%)$ in 2009.

The results of the various scenarios to analyse the relative impact of declining incidence, PMTCT programmes and child treatment show that PMTCT had the largest relative impact. In Botswana the estimated HIV-related under-5 mortality was $380 \%$ higher when analysed without the impact of PMTCT while in Zimbabwe there was an estimated 17\% increase in HIVrelated mortality. When no child ART programmes were implemented, there was a $5 \%$ change in HIV-related mortality in both Botswana and Zimbabwe, while stable HIV incidence among pregnant women resulted in increases of $14 \%$ and $19 \%$ in HIV-related mortality in the two countries. Cotrimoxazole resulted in a $26 \%$ increase in HIV-related mortality in Botswana and a $10 \%$ increase in Zimbabwe (table 1).
Figure 3 Proportion of under-5 mortality attributable to HIV in sub-Saharan Africa, 1990-2009.

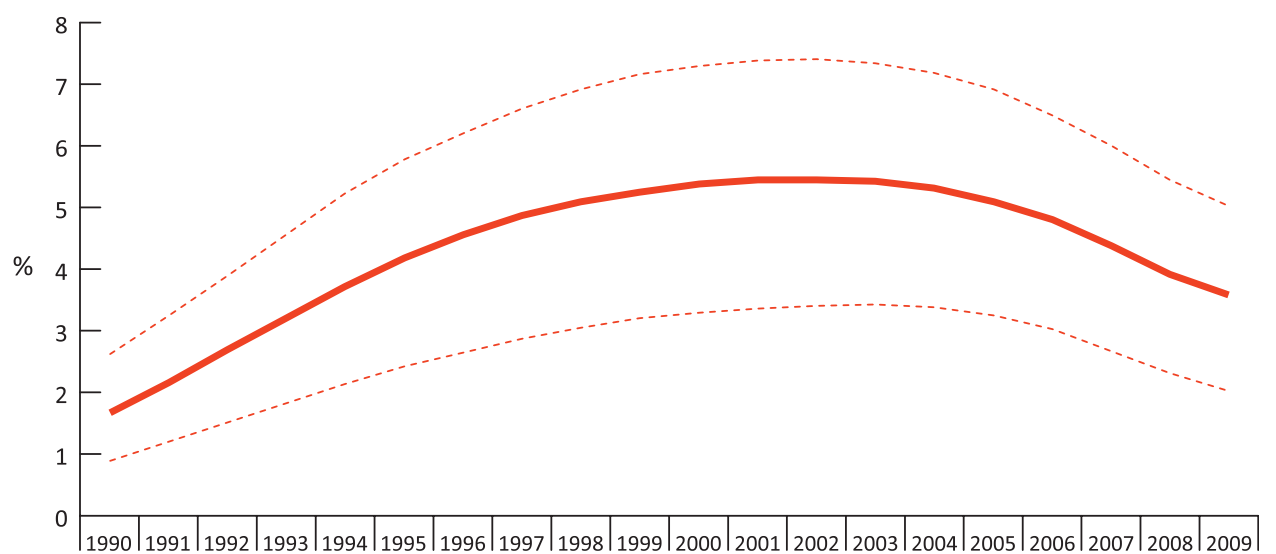


Figure 4 Proportion of under-5 mortality attributable to HIV in African subregions, 1990-2009.
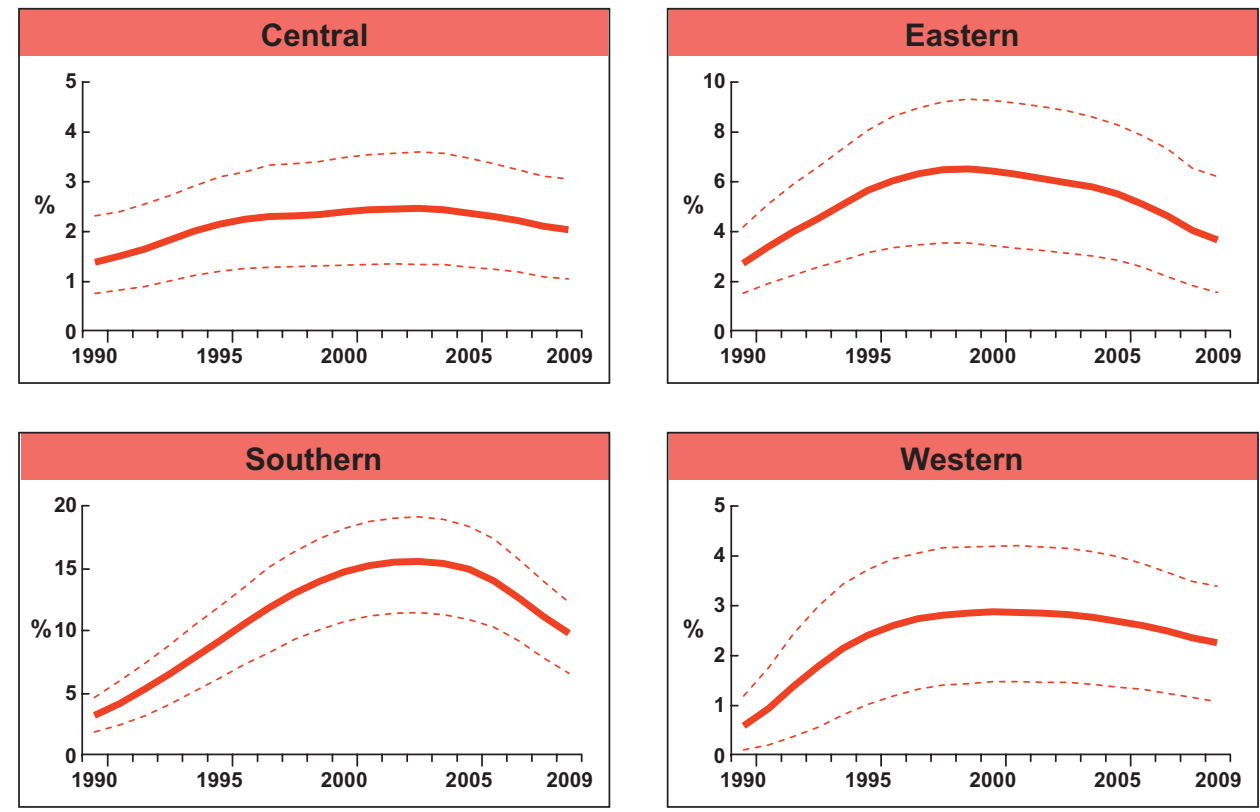

Estimate $\quad$-..-- High and low estimates

\section{DISCUSSION}

The Millennium Development Goal 4 (MDG4) target is to reduce the under- 5 mortality rate by two-thirds between 1990 and 2015. A recent analysis shows that, in all regions of the world including all regions in sub-Saharan Africa, there is evidence of accelerating declines from 2000 to 2010 compared with 1990 to 2000. Within sub-Saharan Africa the rates of decline have increased by more than $1 \%$ in several countries, including some of those most severely affected by HIV such as Botswana, Kenya, Lesotho and Swaziland. ${ }^{6}$

We have explored the contribution of HIV to trends in under-5 mortality by producing estimates of the proportion of under- 5 mortality due to HIV in developing countries, with a specific focus on sub-Saharan Africa, for the period 1990-2009. Globally, in low- and middle-income countries the proportion of deaths due to HIV among children aged $<5$ years increased up to $2.8 \%$ $(1.8-3.8 \%)$ in 2004 and then declined to $2.1 \%(1.2-3.0 \%)$ in 2009. In sub-Saharan Africa the proportion of deaths due to HIV among children aged $<5$ years increased to $5.4 \%(3.3-7.3 \%)$ in $2001-2$ and declined to $3.6 \%(2.0-5.0 \%)$ in 2009 .

Although Nigeria has more under-5 deaths due to HIV than most countries in Southern Africa, the overall proportion of under-5 deaths due to HIV is lower than in those countries. The countries in Southern Africa have lower overall mortality among children aged $<5$ years from other causes.

We also calculated the proportion of HIV-related deaths in a comparable set of 41 countries in sub-Saharan Africa to under5 mortality estimated by the Institute for Health Metrics and Evaluation (IHME). ${ }^{6}$ The overall pattern remains the same, with the peak occurring in 2002 for these countries. The estimated proportions using the IHME denominators are similar with 6.3 in 2002 compared with 5.4 using the Spectrum estimates of total under- 5 deaths. The estimated proportion of HIV-related deaths in 2009 using the IHME estimates of under-5 mortality is 4.3 compared with the estimated proportion of 3.6 using the Spectrum estimates of under-5 mortality.
Figure 5 Number of under-5 deaths attributable to HIV in sub-Saharan Africa, 2009.

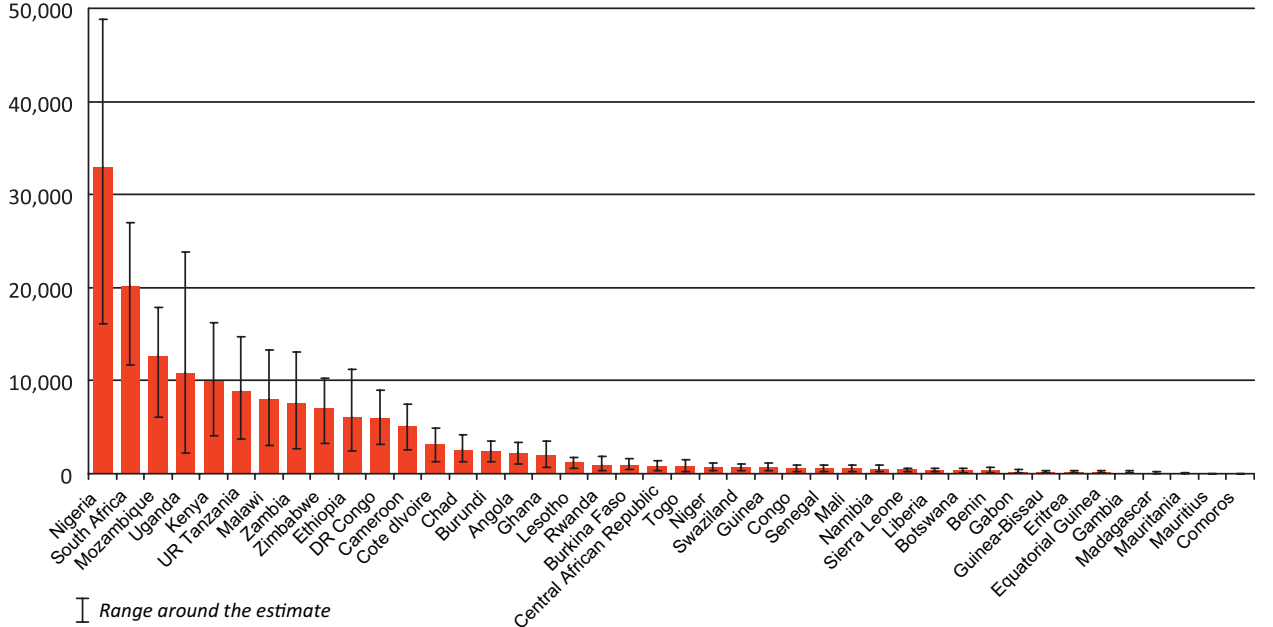


Figure 6 Proportion of under-5 deaths attributable to HIV in sub-Saharan Africa, 2009.

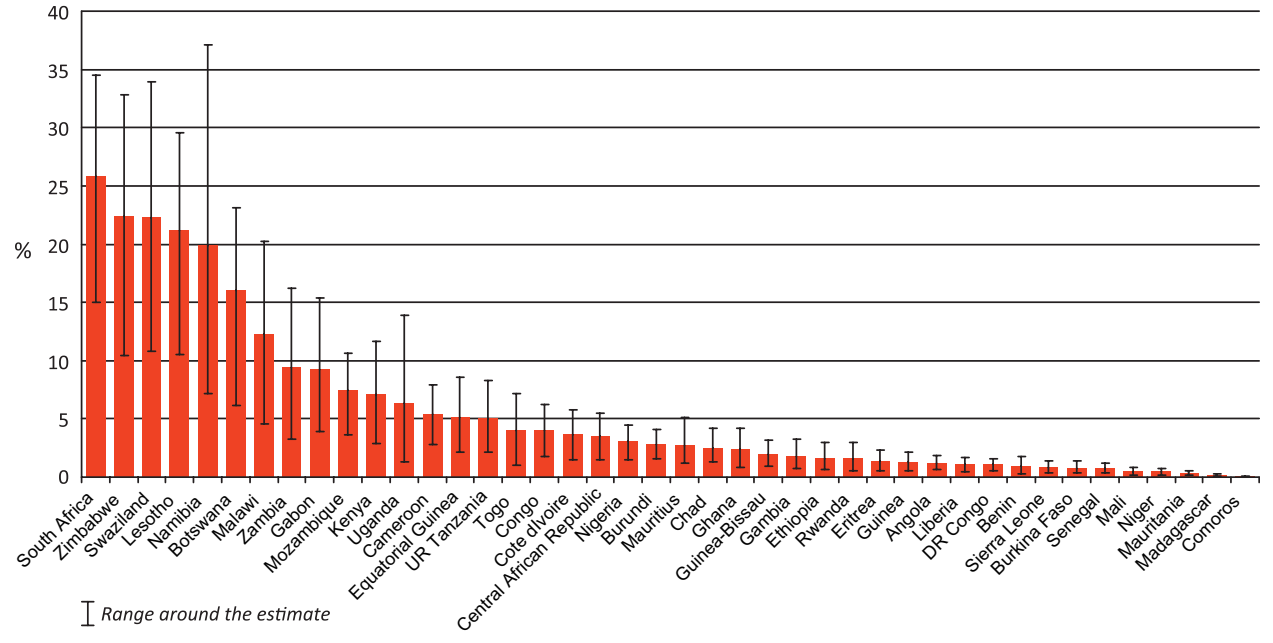

Two previous assessments of the contribution of HIV to under5 mortality showed that HIV was responsible for an increasing proportion of child deaths throughout $2002 .^{21-23}$ The current assessment through 2009 shows a decreasing proportion in the most recent years. The decline in deaths due to HIV can be attributed to a number of factors. During this time period the prevalence of HIV in adults (15-49 years of age) began to decline in several sub-Saharan African countries including Uganda, Kenya, Burkina Faso, Cote d'Ivoire and Zimbabwe. These declines in the adult prevalence of HIV would result in fewer children being born HIV-positive. Since many HIV-positive children die within the first year of life, rapid changes in prevalence among adult women will quickly translate into changes in under-5 mortality. ${ }^{24}$ Also, during this period, progress was made to increase the coverage of maternal ARV agents to prevent mother-to-child transmission-for example, from 10\% to $53 \%$ between 2004 and 2009 in low- and middle-income countries-again resulting in fewer HIV-infected children being born. ${ }^{7}$ 24 In addition, an increasing number of pregnant women are receiving ART for their own health, reducing HIV transmission to their children and lengthening their lives, thereby reducing mortality among their children (both HIV-positive and HIVnegative). ${ }^{25}$ In South Africa, in a largely rural population with a high antenatal HIV prevalence, deaths in infants aged $<2$ years declined by $49 \%$ between 2001 and $2006 .^{26}$ This was attributed to the availability of PMTCT and ART in public health programmes. Finally, during the same period the number of children aged $<14$ years in low- and middle-income countries on ART increased from 75000 in 2005 to 356400 in 2009 (28\% (21-43\%) coverage of children in need of ART), resulting in fewer deaths. ${ }^{7}$

Table 1 Coverage of HIV programmes and impact of changing programme coverage and HIV incidence among women on under-5 mortality

\begin{tabular}{lrc}
\hline & Botswana & Zimbabwe \\
\hline Coverage of programmes & & \\
$\quad$ PMTCT & $990 \%$ & $56 \%$ \\
Child ART & $89 \%$ & $30 \%$ \\
$\quad$ Cotrimoxazole & $60 \%$ & $30 \%$ \\
Scenarios & & \\
Impacts on child mortality due to HIV & $14 \%$ & $19 \%$ \\
Stable incidence & $380 \%$ & $17 \%$ \\
No PMTCT & $5 \%$ & $5 \%$ \\
No ART & $26 \%$ & $10 \%$ \\
No cotrimoxazole &
\end{tabular}

ART, antiretroviral therapy; PMTCT, prevention of mother-to-child transmission of HIV.
The analysis of varying scenarios in two countries with a declining incidence of HIV showed the relatively important impact of PMTCT programmes on under-5 mortality when PMTCT coverage is high. All components, however, contributed child ART on child mortality is similar for Botswana and Zimbabwe with different programme coverage, that coverage is distributed over the entire range of $0-14$ years of age while our measure only reflects children aged $<5$ years. Additionally, many children are not diagnosed in time and may die before being determined to be eligible for ART. Finally, ART scale-up in children has only occurred over the past few years.

HIV infection progresses more aggressively in infants infected during birth and delivery than in adults. Early treatment within the first few months of life can dramatically improve the survival rates of children with HIV. However, many children do not receive treatment as soon as needed; indeed, many children die before it is determined that they are infected with HIV. New WHO guidelines recommend that all infants $<1$ year of age with confirmed HIV infection should start ART, irrespective of the clinical or immunological stage. Also, while PMTCT programmes have been scaled up dramatically in the last 10 years, current levels of coverage are still low. In 2009 over half of the 1.4 million pregnant women living with HIV are estimated to have received ARV drugs to prevent transmission of HIV to their infants. An estimated 53\% (40-57\%) of pregnant women living with HIV received ARV drugs to reduce the risk of transmitting HIV to their infants, up from 45\% (37-57\%) in 2008 and $15 \%(12-18 \%)$ in $2005 .^{7}$ An increased commitment to greater coverage and use of more effective ARV regimens will

\section{Key messages}

- In 2009 HIV accounted for $2.1 \%(1.2-3.0 \%)$ of the under-5 deaths in low- and middle-income countries and $3.6 \%$ (2.0-5.0\%) in sub-Saharan Africa.

- The percentage of under-5 deaths due to HIV has been falling in the last decade.

- The fall in under-5 deaths due to HIV has been driven by a combination of factors, including scale-up of prevention of mother-to-child transmission and treatment for pregnant women and children as well as a decrease in HIV prevalence among pregnant women. to the reduction in under- 5 mortality. Although the impact of 
be needed if we are to continue to reduce child deaths due to HIV.

Funding KS developed and drafted the manuscript; JD provided analytical and data coordination support; JS provided input, comments and developed Spectrum; PA and MM provided comments.

\section{Competing interests None}

Provenance and peer review Not commissioned; externally peer reviewed.

\section{REFERENCES}

1. United Nations. Declaration of Commitment on HIVIAIDS. New York: United Nations, 2001:25-7.

2. Adetunji J. Trends in under-5 mortality rates and the HIV/AIDS epidemic. Bull World Health Organ 2000; 78:1200-6.

3. Murray CJ, Laakso T, Shibuya K, et al. Can we achieve Millennium Development Goal 4 ? New analysis of country trends and forecasts of under- 5 mortality to 2015 Lancet 2007:370:1040-54.

4. Bryce J, Boschi-Pinto C, Shibuya K, et al. WHO estimates of the causes of death in children. Lancet 2005;365:1147-52.

5. Black RE, Cousens S, Johnson HL, et al. Global, regional, and national causes of child mortality in 2008: a systematic analysis. Lancet 2010;375:1969-87.

6. Rajaratnam JK, Marcus JR, Flaxman AD, et al. Neonatal, postneonatal, childhood, and under-5 mortality for 187 countries, 1970-2010: a systematic analysis of progress towards Millennium Development Goal 4. Lancet 2010;375:1988-2008.

7. WHO/UNAIDS/UNICEF. Towards Universal Access: Scaling Up Priority HIVIAIDS Interventions in the Health Sector. Progress Report. Geneva: WHO/UNAIDS/UNICEF, 2010.

8. UNICEF. The State of the World's Children: Child Survival New York: UNICEF, 2008

9. Gregson S, Garnett GP, Nyamukapa CA, et al. HIV decline associated with behavior change in eastern Zimbabwe. Science 2006;311:664-6.

10. Central Statistical Agency (Ethiopia) and ORC Macro. Ethiopia Demographic and Health Survey 2005. Addis Ababa, Ethiopia and Calverton, Maryland, USA: Central Statistical Agency and ORC Macro, 2006.

11. Zambia CSO, Ministry of Health Z, Tropical Diseases Research Centre N, Zambia, Zambia Uo, MEASURE DHS MII. Zambia Demographic and Health Survey 2007. Preliminary Report 2008.
12. Ministry of Health, Kenya. Kenya AIDS Indicator Survey 2007. Nairobi: National AIDS and STI Control Programme, Ministry of Health, Kenya, 2009.

13. Brown T, Salomon JA, Alkema $L$, et al. Progress and challenges in modelling country-level HIV/AIDS epidemics: the UNAIDS Estimation and Projection Package 2007. Sex Transm Infect 2008;84(Suppl 1):i5-10.

14. Stover J, Johnson P, Zaba B, et al. The Spectrum projection package: improvements in estimating mortality, ART needs, PMTCT impact and uncertainty bounds. Sex Transm Infect 2008;84(Suppl 1):i24-30.

15. Lyerla R, Gouws E, Garcia-Calleja JM, et al. The 2005 Workbook: an improved too for estimating HIV prevalence in countries with low level and concentrated epidemics. Sex Transm Infect 2006;82(Suppl 3):iii41-4.

16. Stover J, Johnson P, Hallett T, et al. The Spectrum projection package: improvements in estimating incidence by age and sex, mother-to-child transmission HIV progression in children and double orphans. Sexually Transm Infect 2010;86(Suppl II):ii16-ii21.

17. Mahy M, Lewden C, Brinkhof MWG, et al. Derivation of parameters used in spectrum for eligibility for antiretroviral therapy and survival on antiretroviral therapy. Sexually Transm Infect 2010;86(Suppl II):ii28-ii34.

18. Anon. AIDS Info e-linc. 2010. http://aidsinfo.unaids.org

19. Gregson S, Gonese E, Hallett T, et al. HIV decline in Zimbabwe due to reductions in risky sex? Evidence from a comprehensive epidemiological review. Int J Epidemiol Published Online First: 20 Apr 2010

20. Stover J, Fidzani B, Molomo BC, et al. Estimated HIV trends and program effects in Botswana. PLOS ONE 2008;3:e3729.

21. Walker N, Schwartlander B, Bryce J. Meeting international goals in child survival and HIV/AIDS. Lancet 2002;360:284-9.

22. Newell ML, Brahmbhatt $H$, Ghys PD. Child mortality and HIV infection in Africa: a review. AIDS 2004;18(Suppl 2):S27-34.

23. Newell ML, Coovadia H, Cortina-Borja M, et al. Mortality of infected and uninfected infants born to HIV-infected mothers in Africa: a pooled analysis. Lancet 2004;364:1236-43.

24. Violari A, Cotton MF, Gibb DM, et al. Early antiretroviral therapy and mortality among HIV-infected infants. N Engl J Med 2008;359:2233-44.

25. Zaba B, Whitworth J, Marston M, et al. HIV and mortality of mothers and children: evidence from cohort studies in Uganda, Tanzania, and Malawi. Epidemiology 2005:16:275-80.

26. Ndirangu J, Newell ML, Tanser F, et al. Decline in early life mortality in a high HIV prevalence rural area of South Africa: evidence of HIV prevention or treatment impact? AIDS 2010;24:593-602. 